\title{
On Optimal Input Design for Networked Systems
}

\author{
Per Hägg ${ }^{\mathrm{a}}$, Bo Wahlberg ${ }^{\mathrm{a}}$ \\ ${ }^{a}$ Department of Automatic Control and ACCESS, School of Electrical Engineering, KTH Royal Institute of Technology, \\ SE-100 44 Stockholm, Sweden.
}

\begin{abstract}
The topic of this paper is optimal input signal design for identification of interconnected/networked dynamic systems. We consider the case when it is only possible to design some of the input signals, while the rest of the inputs are only measurable. This is most common in industrial applications, where external excitation can only be applied to some subsystems. One example is feed-forward control from measurable disturbances. The optimal input signal will be correlated with the measured signals. The main purpose of this paper is to reveal how to re-formulate the input design problem for networked systems as an input design problem for feedback control systems. We can then use the powerful partial correlation approach for optimal closed loop input design. This means that the corresponding networked optimal input design problem can be formulated as a semi-definite program, for which there are efficient numerical methods. We evaluate this approach using two numerical examples with important applications. The result reveals some non-trivial interesting properties of the optimal input signals.
\end{abstract}

Key words: System identification, closed-loop identification, interconnected systems, prediction error methods.

\section{Introduction}

System identification is concerned with the estimation and validation of mathematical models of dynamical systems from experimental data. Identification has mainly been studied in a classical open-loop or closedloop setting with inputs, outputs and unmeasurable disturbances. However, networks of interconnected dynamical systems are becoming more and more important in many fields of engineering. A networked system consists of interconnected subsystems, see Fig. 1 for an example. A common objective of the system identification experiment is to model one subsystem or a part of the networked system. The structure of this problem raises many interesting system identification questions.

The first question is how to determine the structure of the underlying network that generated the measurements. Early work addressing this problem can be found in [9] and [1]. Some recent work on topology identification of networked systems can be found in [18], [19], [23]. If the network is sparsely interconnected, regularization ideas could be applied, see [20] and [2] .

In many applications the structure of the network is known but the dynamics of the subsystem building

Email addresses: pehagg@kth.se (Per Hägg), bo@kth.se (Bo Wahlberg).

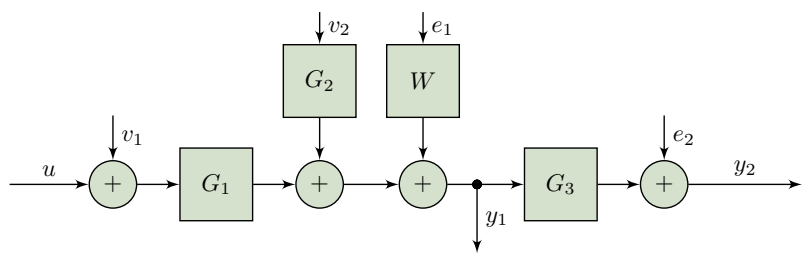

Fig. 1. Example of a network of interconnected subsystems.

up the network are not. The classical Prediction Error Method (PEM), [17], is often applicable in this case. However, identifiability of the subsystems could be an issue. For example, in a cascade of two systems where only the output of the second system is measured, without prior knowledge it is impossible to say which dynamic belongs to which system. In [5] the conditions for consistent identification of closed-loop systems with PEM is extended to the network settings and conditions on the interconnection structure, the presence of noise sources and excitation signals are derived. The followup paper [4] asks which signals must be included in the predictor model to guarantee consistency. Even if the estimate of a model is consistent, the quality of the identified model could be inadequate. Hence, the next question asks what affects the quality of the identified model. Some initial work in this is [21] and [11] where the basic building blocks of a networked system, the cascade and the parallel are analyzed. The effect of sensor placement, input signals and common dynamics 
of the subsystems on the asymptotic properties of the identified models are discussed.

On vital question is how should one design the excitation signal used during the experiment to obtain as much information as possible about the subsystems in the network? Here, we will consider the excitation design problem using the Application Oriented Input Design framework, [15], where the system identification experiment is designed such that the identified system satisfy some application requirements.

However, some special attention is required to apply this framework to networked systems. For example, we need to take into consideration that although some of the signals are measurable we cannot excite them, something that is inherent in many industrial applications. In this case it is possible to correlate the input with the measured disturbances or, equivalently, feed-forward the disturbance to the input. In this paper we will show how to formulate a large class of networked input design problems in this framework and how to formulate the problem as a semi-definite program (SDP) that can be solved efficiently using numerical methods. This paper is a generalization of the preliminary results in [10] where optimal input design for feed-forward control was studied.

The main contributions of this paper include:

- Develop a framework for optimal input design for networked systems, particulary for systems where some of the input signals are measurable but cannot be designed. Furthermore we show how to formulate the optimization problem as a semi-definite program.

- Show how to use the framework in two simulation examples with practical applicability which reveals some interesting properties of the optimal input signals.

\section{Networked Systems}

Consider a networked system in Fig. 2 on the form

$$
y(t)=G_{u}(q) u(t)+G_{v}(q) v(t)+H_{0}(q) e(t),
$$

where $u(t)=\left[u_{1}(t) \cdots u_{p}(t)\right]^{T}$ is the input vector, $v(t)=\left[v_{1}(t) \cdots v_{r}(t)\right]$ is the vector of measurable or known disturbances, $e(t)=\left[e_{1}(t) \cdots e_{m}(t)\right]^{T}$ the unmeasurable disturbances and $y(t)=\left[y_{1}(t) \cdots y_{m}(t)\right]^{T}$ are the measured outputs. The measurement noise is assumed to be zero mean white noise with covariance $\operatorname{diag}\left(\lambda_{1}, \ldots, \lambda_{m}\right)$ while the measurable disturbances are modeled as stationary stochastic processes with known spectral properties, i.e., $v(t)$ can be written as $v(t)=M(q) s(t)$, where $s(t)$ is a zero mean Gaussian process with covariance $\Sigma_{s}$. The spectrum of $v(t)$ can hence be written as $\Phi_{v}(\omega)=M\left(e^{j \omega}\right) \Sigma_{s} M\left(e^{-j \omega}\right)^{T}$. Furthermore we assume that the disturbances are independent of the measurement noise $e(t)$.

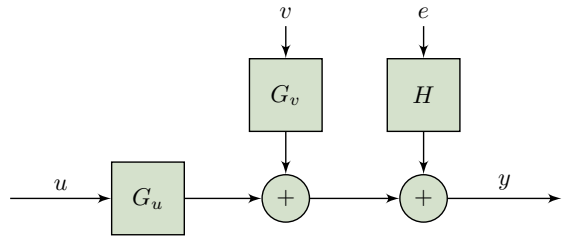

Fig. 2. Considered networked system.

For ease of notation, we will sometimes omit the time or frequency argument when there is no risk of confusion.

We will now give two examples to show that systems of the form (1) can represent a quite broad class of networked systems.

\subsection{Networked System}

The networked system in Fig. 1 can be written on the form (1) as

$$
y=\left[\begin{array}{c}
G_{1} \\
G_{1} G_{3}
\end{array}\right] u+\left[\begin{array}{cc}
G_{1} & G_{2} \\
G_{1} G_{3} & G_{2} G_{3}
\end{array}\right] v+\left[\begin{array}{cc}
W & 0 \\
G_{3} W & 1
\end{array}\right] e .
$$

Here we can only design one of the inputs, namely $u$, while $v_{1}$ and $v_{2}$ are given from the application. This is a common setup in many industrial problems. The goal could be to identify the subsystems $G_{1}(q)$ and $G_{3}(q)$ for a subsequent control design. The question is then how should we excite the system so that this identification is as good as possible, taking into account the known properties and the measurements of the disturbances $v$ ?

\subsection{Reference Feed-Forward}

Consider the feedback system in Fig. 3.

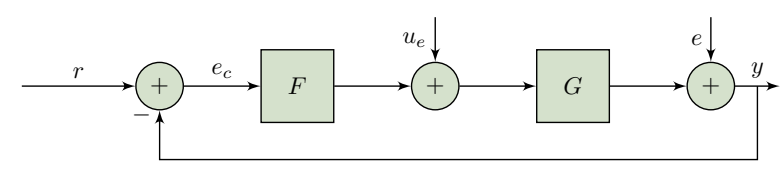

Fig. 3. Feedback with excitation

The system operates in a closed loop with a given controller $F(q)$ and a given reference signal $r(t)$. For example, we want to estimate the system $G(q)$ to re-tune the controller $F(q)$. During the system identification experiment we can excite the system by $u_{e}$ while still running the system in a closed loop with a given reference, so that a minimum of production is lost during the experiment. The objective could then be to minimize the output variance during the experiment, i.e., to disturb the process as little as possible during the experiment while the identified model satisfies some quality constraints. Since the reference $r(t)$ is known we can correlate the 
input $u_{e}$ with $r$ using, for example, a feed-forward controller. The closed-loop system can be written on the form (1) as

$$
y(t)=\frac{G}{1+G F} u_{e}(t)+\frac{G F}{1+G F} r(t)+\frac{1}{1+G F} e(t) .
$$

\section{System Identification}

The goal is to identify the dynamics of the subsystems in the network using a Prediction Error Method [17].

The model set is given by $G_{u}(q, \theta), G_{v}(q, \theta), H(q, \theta)$, where $\theta \in R^{n}$ is the model parameter vector that we want to estimate and we assume that the true system can be described by the model with a parameter vector denoted $\theta_{0}$. We will also assume that the identifiability conditions in [5] are satisfied so that the system is identifiable and can be consistently identified.

We denote the model parameter vector estimated with a Prediction Error Method from $N$ data points of the inputs, measurable disturbances and outputs by $\hat{\theta}_{N}$. Under some mild assumptions, the asymptotic (in $N$ ) covariance matrix of the estimated parameters

$$
P=\lim _{N \rightarrow \infty} N E\left(\hat{\theta}_{N}-\theta_{0}\right)\left(\hat{\theta}_{N}-\theta_{0}\right)^{T}
$$

can be written as

$$
\begin{aligned}
P^{-1} & =\sum_{k=1}^{p} \frac{1}{2 \pi \lambda_{k}} \int_{-\pi}^{\pi} \mathcal{F}_{k}\left(e^{j \omega}\right) \Phi_{\chi_{0}}(\omega) \mathcal{F}_{k}^{*}\left(e^{j \omega}\right) d \omega \\
& +\frac{1}{2 \pi} \sum_{k=1}^{p} \int_{-\pi}^{\pi} \mathcal{H}_{k}\left(e^{j \omega}\right) \mathcal{H}_{k}^{*}\left(e^{j \omega}\right) d \omega
\end{aligned}
$$

where $\Phi_{\chi_{0}}(\omega)$ is the spectrum of $\chi_{0}(t)=\left[u(t)^{T}, s(t)^{T}\right]^{T}$,

$$
\Phi_{\chi_{0}}(\omega)=\left[\begin{array}{cc}
\Phi_{u}(\omega) & \Phi_{u s}(\omega) \\
\Phi_{s u}(\omega) & \Sigma_{s}
\end{array}\right]
$$

and where the $l$-th row of the matrix $\mathcal{F}_{k}(q)$ is given by the $k$-th row of the matrix

$$
\left.H^{-1}(q, \theta)\left[\frac{\partial G_{u}(q, \theta)}{\partial \theta_{l}} \frac{\partial G_{v}(q, \theta)}{\partial \theta_{l}} M(q)\right]\right|_{\theta=\theta_{0}},
$$

and $\mathcal{H}_{k}$ is defined similarly but for the matrix $\frac{\partial H(q, \theta)}{\partial \theta_{l}}$. Furthermore, asymptotically, the identified parameter vector $\hat{\theta}_{N}$ lies within the set

$$
\mathcal{E}_{S I}=\left\{\theta: \frac{1}{2}\left[\theta-\theta_{0}\right]^{T} P^{-1}\left[\theta-\theta_{0}\right] \leq \frac{\kappa_{\alpha}}{N}\right\}
$$

with probability $\alpha,[17]$. The constant $\kappa_{\alpha}$ can be determined from the $\chi^{2}$-distribution as a function of the probability $\alpha$ and the number of estimated parameters.

\section{Input Design for Interconnected Systems}

From (3) it is seen that the variance of the parameters is affected by the signal spectrum. Thus, by exciting the system with a suitable spectrum we can influence the quality of the identified models. In the setting considered here we can control the inputs $u(t)$ and consequently design the spectrum $\Phi_{u}(\omega)$. But since we can measure $v(t)$ (and consequently $s(t)$ ) we can correlate the input signal with the measurable disturbances and thus also influence $\Phi_{u s}(\omega)$.

We define the experimental cost function $f_{\text {cost }}\left(\Phi_{\chi_{0}}(\omega)\right)$ that we we would like to minimize during the experiment. This could for example be minimum input or output variance. For simplicity, we require the objective function to be a convex function in the decision variable. This covers many common cost functions in input design.

We can now formulate the optimal input design as the following optimization problem

$$
\begin{array}{cl}
\underset{\Phi_{\chi_{0}}(\omega)}{\operatorname{minimize}} & f_{\text {cost }}\left(\Phi_{\chi_{0}}(\omega)\right) \\
\text { subject to } & \varepsilon_{S I} \in \varepsilon_{a p p} \\
& \Phi_{\chi_{0}}(\omega) \succeq 0, \quad \forall \omega
\end{array}
$$

where the optimization variable is the parts of the spectrum that we can affect, c.f., (4). We assume for now that $\varepsilon_{S I} \in \varepsilon_{a p p}$ is a convex constraint on the quality of the identified parameters. We will explain how to choose this set in Section 5. The last constraint ensures that the spectrum is realizable.

While the optimization problem (6) is convex, it is still hard to solve due to the optimization over the infinite dimensional spectrum, $\Phi_{\chi_{0}}(\omega), \omega \in[-\pi, \pi]$. The main contribution in this paper is to find a finite dimensional parametrization of the spectrum so that the optimization problem (6) is convex in these parameters and suitable for networked systems.

Two common ways to parameterize the spectrum in the open-loop case is to use a finite dimensional parametrization [16] or a partial correlation parametrization [7]. Here we will focus on the latter. We introduce the generalized moments of the spectrum

$$
m_{k}=\frac{1}{2 \pi} \int_{-\pi}^{\pi} \frac{1}{\left|D\left(e^{j \omega}\right)\right|^{2}} \Phi_{\chi_{0}}(\omega) e^{j k \omega} d \omega
$$

where $D(z)=\sum_{l=0}^{m} d_{l} z^{l}$ is such that the coefficients are real and obey $d_{m} \neq 0$ and has all its roots outside the 


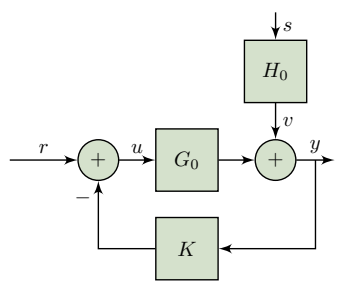

(a) Feedback

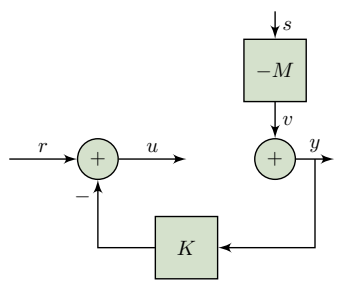

(b) Feed-forward
Fig. 4. The relation between the input $u(t)$ in the closed loop case and the feed-forward case.

closed unit disc. By a suitable choice of this polynomial, many input design problems can be expressed as affine functions of a finite number of generalized moments, see for example $[7,22]$.

The idea of the partial correlation approach is to find conditions on these moments that guarantee the existence of an infinite extension so that the complete sequence defines a spectrum. It can be shown that such an extension exists if the block Toeplitz matrix

$$
T_{n}=\left[\begin{array}{ccc}
m_{0} & \cdots & m_{n}^{T} \\
\vdots & \ddots & \vdots \\
m_{n} & \cdots & m_{0}
\end{array}\right]
$$

is positive semi-definite [7].

This approach is, however, not directly applicable to our problem. The problem is that we do not have control over the disturbance $v(t)$. The partial correlation approach only guarantees that an extension exists, not that the extension exactly corresponds to the given properties of the external signals $v(t)$. It would be applicable if we were allowed to also design $v(t)$. However, the partial correlation approach has been extended to closed-loop optimal input design [14] and recently to the MIMO case in [12] and [13]. We will show that the problem considered in this paper can be seen as a non-trivial special case of the closed-loop optimal input design problem.

Consider the closed-loop setup in Fig. 4a. In closed-loop input design the objective is to design the input $u(t)$ to use during the experiment. To this end, the controller $K(q)$ and the spectrum of the reference signal $r(t)$ is designed such that $u(t)=-K(q) y(t)+r(t)$. We can either work with the spectrums $\Phi_{u}$ and $\Phi_{u v}$ or $K$ and $\Phi_{r}$ since there is a one-to-one relation between them.

Consider now the setting in Fig. 4b. Here we want to find conditions such that $\Phi_{u}$ and $\Phi_{u v}$ corresponds to a realizable experiment, or equivalently find $K$ and $\Phi_{r}$ that corresponds to a realizable experiment. From a signal perspective, we see that the considered problem here in Fig. 4b corresponds to the closed-loop case in Fig. 4a when $G_{0}=0$ and $H_{0}=-M$ and where the disturbance $v(t)$ plays the role of the measurement noise in the closed-loop case. We summarize this observation in the following proposition.

Proposition 1 If $G_{0}=0$ and $H_{0}=-M$ in the closed loop setup in Fig. $4 a$,

$$
u(t)=\left(I+K G_{0}\right)^{-1} r(t)-\left(I+K G_{0}\right)^{-1} K H_{0} s(t),
$$

then the signal properties of the input signal correspond to the feed-forward case

$$
u(t)=r(t)+K M s(t)=r(t)+K v(t) .
$$

Consequently, given a controller $K(q)$ and a spectrum $\Phi_{r}(\omega)$ the corresponding $\Phi_{u}(\omega)$ and $\Phi_{u s}(\omega)$ defines a realizable experiment.

Hence, we can use the existing results from closed-loop optimal input design to guarantee that the spectra in the optimization can be realized by a reference signal with spectrum $\Phi_{r}$ and a, in this case, feed-forward controller $K(q)$ with $u(t)=-K(q) y(t)+r(t)=K(q) v(t)+r(t)$.

We will now show how the partial correlation approach from the closed-loop optimal input design [13] can be used in this setting. Again we define the generalized moments as in (7). Note that $m_{k}$ is real and that $m_{-k}=m_{k}^{T}$. If the model structures of $G_{u}(q, \theta), G_{v}(q, \theta)$ and $H(q, \theta)$ are rational, then by choosing $D(z)$ as the least common denominator of $\mathcal{F}_{k}$ defined in (5), $P^{-1}$ is affine in the generalized moments $m_{0}, m_{1}, \ldots, m_{n}$ c.f., (3), see [16]. Many common cost functions in input design, for example minimum input variance, can also be written as linear functions of the generalized moments, see [12].

The input design problem (6) can hence be written as an SDP in a finite number of generalized moments (7). Now we want to classify the set of all sequences $m_{0}, \ldots, m_{n}$ that corresponds to a realizable experiment design, i.e., that corresponds to a $K(q)$ and $\Phi_{r}(\omega)$ that are realizable, or equivalently, that implies that $\Phi_{\chi_{0}}(\omega) \succeq 0$ for all $\omega$. In [13] a semi-definite description of this set is given. We will briefly summarize the result here.

Partition the sequence $\left(m_{0}, m_{1}, \ldots, m_{n}\right)$ of $(p+r) \times$ $(p+r)$ matrices $m_{-k}=m_{k}^{T}$ into 4 blocks $m_{k, 11}, m_{k, 12}$, $m_{k, 21}, m_{k, 22}$ corresponding to the partition of $\Phi_{\chi_{0}}$ into $\Phi_{u}, \Phi_{u s}$ and $\Sigma_{s}$. Then the sequence $\left(m_{0}, m_{1}, \ldots, m_{n}\right)$ is extendable with $\left(m_{n+1}, m_{n+2}, \ldots\right)$ and defines a valid experiment if the following conditions hold

(1) $m_{k, 22}=\frac{1}{2 \pi} \int_{-\pi}^{\pi} \frac{\Sigma_{s}}{\left|D\left(e^{j \omega}\right)\right|^{2}} e^{j k \omega}$ for $k=-n, \ldots, n$.

(2) $\sum_{l=0}^{m} d_{l} m_{k-l, 21}=0$ for $k=1, \ldots, n$.

(3) The symmetric Toeplitz matrix $T_{n}$ defined in (8) is positive semi-definite.

A proof can be found in [13]. 


\subsection{Generating the Input Signal}

Solving the optimization problem gives the optimal generalized moments $m_{0}, m_{1}, \ldots, m_{n}$. The problem is then to find the feed-forward controller $K$ and the spectrum $\Phi_{r}$ from these matrices. In the case when the optimal $T_{n}$ is positive definite the spectrum can be recovered by

$$
\Phi_{\chi_{0}}(\omega)=\left|D\left(e^{j \omega}\right)\right|^{2} A\left(e^{j \omega}\right)^{-*} A(0) A\left(e^{j \omega}\right)^{-1}
$$

where $A(z)=U(z) T_{n}^{-1} U^{T}(0)$ and $U(z)=\left[z^{n} I \cdots z^{0} I\right]$. The input spectrum $\Phi_{u}(\omega)$ and the cross spectrum $\Phi_{u s}(\omega)$ can then be recovered from the corresponding blocks of $\Phi_{\chi_{0}}(\omega)$. Finally the controller $K\left(e^{j \omega}\right)$ and the spectrum of the exogenous input $\Phi_{r}$ can be found from

$$
\begin{aligned}
K\left(e^{j \omega}\right) & =\Phi_{u s}(\omega) M\left(e^{j \omega}\right)^{-1} \Sigma_{s}^{-1}, \\
\Phi_{r}(\omega) & =\Phi_{u}(\omega)-K\left(e^{j \omega}\right) M\left(e^{j \omega}\right) \Sigma_{s} M^{*}\left(e^{j \omega}\right) K^{*}\left(e^{j \omega}\right) .
\end{aligned}
$$

If $T_{n}$ is singular, the calculations become more involved and are omitted here. See [13] for complete details on how to recover the controller and the spectrum in this case.

\section{Application Oriented Input Design}

The objective of the optimal input design is to design an input so that the experiment gives estimates of the parameters $\theta$ of a certain accuracy. We will use the application oriented input design framework in [15]. The idea is to define the set of acceptable parameters based on the intended use of the model.

We let $V_{a p p}(\theta)$ denote an application cost that measures the degradation in performance due to model errors when the identified model is used in the application. Assume that we have an upper limit on the performance degradation allowed in our application, i.e., that $V_{a p p}(\theta) \leq \frac{1}{\gamma}$ where $\gamma$ is a positive constant, then the goal of the system identification is to find an estimate of the parameter vector so that the identified parameters satisfies $V_{a p p}\left(\hat{\theta}_{N}\right) \leq \frac{1}{\gamma}$.

This set of all parameters that satisfies this inequality can be approximated by [15]

$$
\mathcal{E}_{a p p}=\left\{\theta: \frac{1}{2}\left[\theta-\theta_{0}\right]^{T} V_{a p p}^{\prime \prime}\left(\theta_{0}\right)\left[\theta-\theta_{0}\right] \leq \frac{1}{\gamma}\right\} .
$$

To satisfy the application constraint we need to ensure that the identified parameters lie within the application set (9) with high probability, i.e., that $\mathcal{E}_{S I} \subseteq \mathcal{E}_{\text {app }}$. This is equivalent to

$$
\frac{N}{\kappa} P^{-1} \succeq \gamma V_{a p p}^{\prime \prime}\left(\theta_{0}\right)
$$

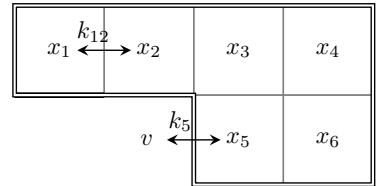

Fig. 5. A building with 6 rooms illustrating two of the heat transfer coefficients $k_{12}$ and $k_{5}$.

where $A \succeq B$ means that $A-B$ is positive semi-definite. If the above LMI holds then $\hat{\theta} \in \mathcal{E}_{\text {app }}$ with at least probability $\alpha$. Hence, the LMI constraint (10) is included in the optimization problem (6).

\subsection{The Unknown True System}

To find the optimal input signal the true parameters of the system, $\theta_{0}$, are needed. This dilemma appears in most optimal input design problems. A common solution to this is to use adaptive or iterative input design. From an initial experiment or prior knowledge of the system, an initial design is performed and the parameters are identified. As more information from the system is available the input signal properties are changed, see e.g., [6].

\section{Simulation Examples}

In this section we will show how the framework for input design for interconnected systems can be applied to two simulation examples.

\subsection{Building Temperature Control}

The first example considers the problem of controlling the temperature in a building using radiators. The model of the system we will use is a simplified version of the one given in [8] but extended to the MIMO case.

The control signals, $u_{i}(t)$, are the temperature of the radiator in each room, and the state $x_{i}(t)$ is the temperature in room $i$ that we would like to control. The temperature in room $i$ is also influenced by the outside temperature, $v(t)$, due to heat transfer in the walls with heat transfer coefficient $k_{i}$. Furthermore, the change in temperature in one room also depends on the temperature in neighboring rooms due to heat transfer. The heat transfer coefficient between room $i$ and $j$ is denoted $k_{i j}=k_{j i}$ and is zero if the two rooms are not adjacent to each other. An example building is illustrated in Fig. 5. A thermometer is fitted outdoors so we assume that we can measure the temperature outside perfectly. The system can be written as

$$
\begin{aligned}
x_{i}(t+1) & =x_{i}(t)+\sum_{j=1}^{p} k_{i j}\left(x_{j}(t)-x_{i}(t)\right) \\
& +k_{i}\left(v(t)-x_{i}(t)\right)+c_{i} u_{i}(t), \\
y_{i}(t) & =x_{i}(t)+e_{i}(t), \quad i=1, \ldots, p
\end{aligned}
$$


where $e_{i}(t)$ is white independent measurement noise.

To illustrate how to apply the proposed input design framework and to simplify the notation, we will consider the case when the building consists of two rooms, i.e., $p=2$. Furthermore, we assume that the outside temperature $v(t)$ can be modeled as white noise with variance $\lambda_{v}$. This assumption is unrealistic in practice since the temperature often is highly correlated but it will allow us to gain some insight into the problem.

The control application objective is to design a feedforward filter, $F_{f}(q)$, to compensate for the changing outdoor temperature, i.e., $u(t)=F_{f}(q) v(t)$. One can show that the optimal feed-forward filter that gives zero error due to changing outside temperature is given by

$$
F_{f}(q)=-\left[\frac{k_{1}}{c_{1}} \frac{k_{2}}{c_{2}}\right]^{T}
$$

However, the true parameters, $\theta=\left[k_{1}, k_{2}, k_{12}, c_{1}, c_{2}\right]^{T}$ of the system are unknown and need to be identified. The goal of the system identification experiment is to identify the parameters with such accuracy that the feedforward filter based on these parameters keeps the variance in the indoor temperature due to changing outdoor temperature less than $1 / \gamma$, i.e., the application cost is

$$
V_{a p p}(\theta)=E\left\{\left\|\left(G_{v}+G_{u} F_{f}(\theta)\right) v(t)\right\|_{2}^{2}\right\} \leq \frac{1}{\gamma}
$$

where $G_{u}$ and $G_{v}$ are the transfer functions of the true system to output $y(t)$ from the input $u(t)$ and disturbance $v(t)$, respectively.

We will now use the framework outlined in Section 4 to formulate an optimal experiment for this case. The least common denominator of the gradients $\mathcal{F}_{i}$ defined in Section 3 is

$$
D(z)=\left(\left(1-z\left(1-k_{1}-k_{12}\right)\right)\left(1-z\left(1-k_{2}-k_{12}\right)\right)-k_{12}^{2} z^{2}\right)^{2} .
$$

By defining the generalized moments $m_{k}$ as in (7) the inverse parameter matrix, $P^{-1}$ can be written as a linear combination of $m_{k}, k=-4, \ldots, 4$.

The signal energy of $u_{i}$ can be written in terms of $m_{k}$ as

$$
\begin{aligned}
\frac{1}{2 \pi} \int_{-\pi}^{\pi} \Phi_{u}^{(i)}(\omega) d \omega & =\frac{1}{2 \pi} \int_{-\pi}^{\pi} \frac{\left|D\left(e^{j \omega}\right)\right|^{2}}{\left|D\left(e^{j \omega}\right)\right|^{2}} \Phi_{u}^{(i)}(\omega) d \omega \\
& =\sum_{k=-4}^{4} \delta_{k}\left[m_{k, 11}\right]_{i i}
\end{aligned}
$$

where $\delta_{k}$ are the coefficients of $\left|D\left(e^{j \omega}\right)\right|^{2}$, i.e., $\left|D\left(e^{j \omega}\right)\right|^{2}=$ $\sum_{k=-4}^{4} \delta_{k} e^{j \omega k}$. The total input energy is then the sum of the individual input energies. In a similar, but more

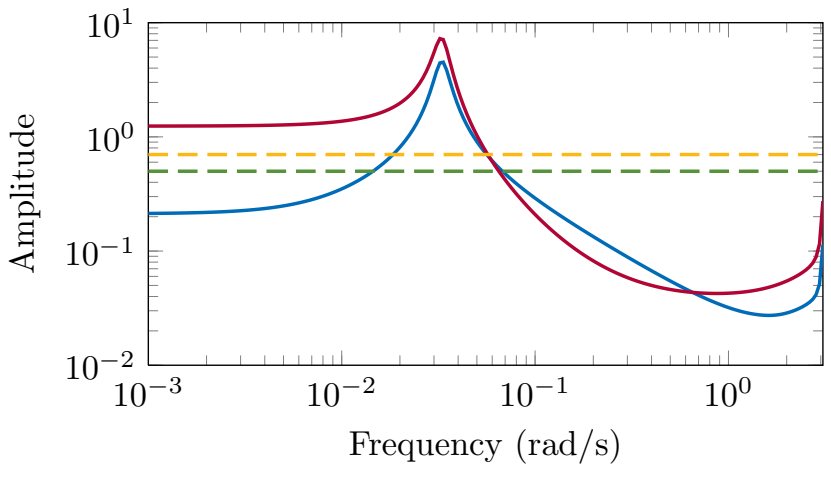

Fig. 6. The magnitude of the optimal feed-forward filters, $K$, to be used during the identification for the minimum input variance case from $v$ to $u_{1}(-)$, from $v$ to $u_{2}(\square)$ and the minimum variance output case from $v$ to $u_{1}(--)$ and $v$ to $u_{2}(--)$.

involved, way it is possible to write the output variance during the experiments as a linear combination of the generalized moments.

We can now formulate the optimal input design problem where we want to find either a minimum variance input signal (save power) or an input signal that gives the lowest output variance (best comfort) while satisfying the application constraints with high probability. The optimization becomes

$$
\begin{array}{ll}
\underset{m_{k}, k=-4, \ldots, 4}{\operatorname{minimize}} & \text { input or output energy } \\
\text { subject to } & \frac{N}{\kappa} P^{-1} \succeq \gamma V_{a p p}^{\prime \prime}\left(\theta_{0}\right) \\
& m_{k, 22}=\frac{1}{2 \pi} \int_{-\pi}^{\pi} \frac{\lambda_{v}}{\left|D\left(e^{j \omega}\right)\right|^{2}} e^{j k \omega}, \forall k \\
& \sum_{l=0}^{4} d_{l} m_{k-l, 21}=0, k=1, \ldots, 4 \\
& T_{4} \succeq 0 .
\end{array}
$$

These two problems are SDPs and can be solved efficiently using numerical methods. The following system parameters will be used: $k_{1}=0.5, k_{2}=0.7, k_{12}=0.3$, $c_{1}=c_{2}=1, \lambda_{v}=1$ and $\lambda_{e}=1$. The experimental conditions are $N=1500, \gamma=100$ and $\kappa=11.07$ corresponding to at least $95 \%$ probability that the identified model satisfies the application requirement. The two problems are solved with CVX, a package for specifying and solving convex programs [3], giving the optimal $m_{k}$, $k=-4, \ldots, 4$. In both cases the optimal $T_{n}$ is singular and we use the results from [13] to recover the feedforward controller and the reference spectrum, $\Phi_{r}$ to be used during the experiment. This gives us $\Phi_{r}(\omega)=0$ and it is therefore enough to use $u(t)=K(q) v(t)$ during the identification. The magnitude of the optimal feedforward controllers $K(q)$ for both problems is shown in Fig. 6. 
We see that when we minimize the input variance, the optimal $K$ is a bandpass filter, while for the output variance case the optimal $K$ is a constant $K=[0.5,0.7]^{T}$. The case when $K=[0.5,0.7]^{T}$ corresponds to the optimal feed-forward controller defined in (11) that gives zero output variance in $y(t)$ due to the measurable disturbance $v(t)$. Using this feed-forward filter during the identification gives $y_{i}(t)=e_{i}(t)$; therefore, we cannot infer anything about the parameters $k_{1}, k_{2}, k_{12}, c_{1}, c_{2}$ from the measurements. However, since we know that the subsystems have the same dynamics, this information is enough to identify the ratio $k_{i} / c_{i}$ which is all information we need to design our feed-forward controller!

For the minimum output variance case the output variance is zero when using the optimal feed-forward controller during the experiment. Without correlating the input with the disturbance, i.e., using $K=0$ during the experiment, the optimal cost is 1.56 . Hence, in this case it is beneficial to also include the knowledge of the disturbance in the input design. The total variance of the optimal minimum variance input signals is 0.23 . Solving the problem without correlating the input with the disturbance gives basically the same cost. In this case, not much is gained by correlating the input with the measurable disturbance during the experiment. But one should note that the results can never be worse when also taking into account that we can correlate the input $u$ with the measurable disturbance $v$.

\subsection{Reference Feed-forward}

Consider the feedback system with excitation in Section 2.2 with

$$
G(z)=\frac{1+b z^{-1}}{1+a z^{-1}}, \quad F(z)=K
$$

The measurement noise $e(t)$ is zero mean white noise with unit variance and the reference signal can be modeled as

$$
r(t)=\frac{1+c}{1+c q^{-1}} s(t) \triangleq M(q) s(t)
$$

where $s(t)$ is zero mean white noise with unit variance.

The objective in this example is to identify the parameters $\theta=[b, a]^{T}$ so that they satisfy $V_{a p p}(\theta)=\left(b-b_{0}\right)^{2}+$ $\left(a-a_{0}\right)^{2} \leq \frac{1}{\gamma}$, where $a_{0}$ and $b_{0}$ are the parameters of the true system. To this end, we want to design a system identification experiment running in closed-loop so that the variance of the control error $e_{c}(t)$ is minimized while the identified parameters satisfy the application constraint. We excite the system by the input $u_{e}(t)$ in Fig. 3 and since the reference $r(t)$ is known we can correlate the input with the reference. Here we can see the reference as the measurable disturbance. The system can be written in the form (1) by using (2).

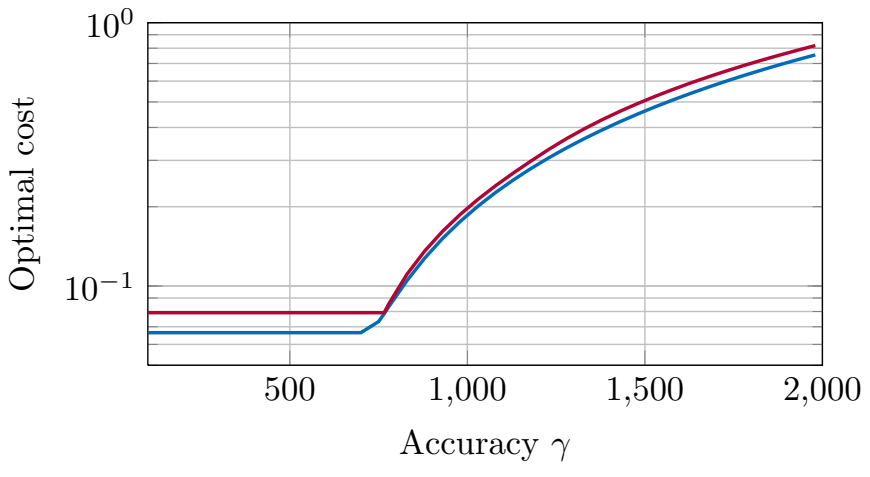

Fig. 7. The optimal cost as a function of the accuracy $\gamma$ when $u$ is correlated ( - ) and uncorrelated ( -$)$ with $r(t)$.

The control error $e_{c}(t)$ can be written as

$$
e_{c}(t)=\frac{1}{1+F G} r(t)-\frac{G}{1+F G} u_{e}(t)-\frac{1}{1+F G} e(t)
$$

and the variance that we want to minimize is given by

$$
f_{\text {cost }}\left(\Phi_{\chi_{0}}\right)=\frac{1}{2 \pi} \int_{-\pi}^{\pi} \frac{1}{|1+F G|^{2}}\left[\begin{array}{ll}
-G & 1
\end{array}\right] \Phi_{\chi_{0}}\left[\begin{array}{c}
-G^{*} \\
1
\end{array}\right] d \omega
$$

where we neglected terms depending on $e(t)$ since both $r(t)$ and $u_{e}(t)$ is independent of $e(t)$.

This optimization problem can now be expressed as an SDP using the framework in Section 4. Again we solve the problem with CVX with the following settings: $a_{0}=$ $-0.8, b_{0}=1.4, c=-0.5, K=3, N=1000$ and $\kappa=5.99$ corresponding to $95 \%$ probability that the application constraint is satisfied. The accuracy $\gamma$ is varied between 100 and 2000 and the optimal cost $f_{\text {cost }}\left(\Phi_{\chi_{0}}\right)$ is shown in Fig. 7. For comparison the optimal solution when we do not correlate $u_{e}(t)$ with the reference $r(t)$ is also shown.

The variance of the control error is about $10 \%$ lower for almost all $\gamma$ when we correlate the input with the reference. For $\gamma \lesssim 700$ the cost is independent of $\gamma$. For low accuracy the application cost is satisfied when the closed-loop system is excited by the measurement noise only. Hence, in the case when $u$ is uncorrelated with $r$, the optimal input that minimizes the control error is $u(t)=0$. In the correlated case it can be shown that the feed-forward controller used, i.e., $u_{e}(t)=F_{f}(q) r(t)$, corresponds to the optimal $\mathrm{H}_{2}$ controller that minimizes

$$
\min _{F_{f}}\left\|\frac{M}{1+F G}-\frac{G F_{f}}{1+F G}\right\|_{2}^{2} .
$$

If $G(z)$ is minimum phase and proper then the optimal feed-forward filter is $F_{f}(q)=M(q) / G(q)$ which gives zero cost. However, in the considered case $G(q)$ has a non-minimum phase zero and the cost is non-zero. 
For higher accuracy (higher $\gamma$ ) the excitation using the optimal $\mathrm{H}_{2}$ feed-forward controller is no longer enough and the variance of the control error has to be increased.

\section{Conclusions}

The objective of this study has been to present a framework for application oriented input design in networked or interconnected systems. Often it is not feasible or it is very expensive to measure every signal in the network or to excite everywhere within a network. This framework could be used to a priori find where to excite or measure by applying this framework and comparing the cost function related to the experiment, $f_{\text {cost }}\left(\Phi_{\chi_{0}}\right)$, for the different configurations of the network.

Future work could extend the framework to the case when there is a correlation between the measurement noise $e$ and the measured disturbance $v$, i.e., feedback is present from the output to the disturbance. In addition to the feed-forward controller, one could also design a feedback controller and hence also correlate the input with the noise, further improving the results. Here only causal feed-forward controllers from the disturbance were considered. In many applications, for example in the reference feed-forward example, the future disturbance could be known, allowing for non-causal controllers. It would be interesting to see if this framework could be extended to also cover this case.

\section{Acknowledgements}

This work was partially supported by the European Research Council under the European Communitys Seventh Framework Programme (FP7/2007- 2013) / ERC Grant Agreement No. 267381, the Swedish Research Council and the Linnaeus Center ACCESS at KTH.

\section{References}

[1] P. Caines. Weak and strong feedback free processes. Automatic Control, IEEE Transactions on, 21(5):737 - 739, October 1976.

[2] Alessandro Chiuso and Gianluigi Pillonetto. A bayesian approach to sparse dynamic network identification. Automatica, 48(8):1553 - 1565, 2012.

[3] Inc. CVX Research. CVX: Matlab software for disciplined convex programming, version 2.0 beta. http://cvxr.com/cvx, September 2012.

[4] Arne Dankers, Paul M.J. Van den Hof, Xavier Bombois, and Pet. Identification of dynamic models in complex networks with prediction error methods - predictor input selection. IEEE Transactions on Automatic Control, 2013. Submitted.

[5] Paul M.J. Van den Hof, Arne Dankers, Peter S.C. Heuberger, and Xavier Bombois. Identification of dynamic models in complex networks with prediction error methodsbasic methods for consistent module estimates. Automatica, 49(10):2994 - 3006, 2013.
[6] L. Gerencsér and H. Hjalmarsson. Adaptive input design in system identification. In Decision and Control, 2005 and 2005 European Control Conference. CDC-ECC '05. 44th IEEE Conference on, pages 4988 - 4993, dec. 2005.

[7] Michel Gevers, Xavier Bombois, Roland Hildebrand, Gabriel Solari, et al. Optimal experiment design for open and closedloop system identification. Communications in Information and Systems, 11(3):197, 2011.

[8] T. Glad and L. Ljung. Control Theory. Control Engineering. Taylor \& Francis, 2000.

[9] C. W. J. Granger. Investigating causal relations by econometric models and cross-spectral methods. Econometrica, 37(3):pp. 424-438, 1969.

[10] Per Hägg and Bo Wahlberg. On optimal input design for feed-forward control. In Decision and Control, 2013. CDC 2013. 52nd IEEE Conference on, 2013.

[11] Per Hägg, Bo Wahlberg, and Henrik Sandberg. On identification of parallel cascade serial systems. In Proceedings of the 18th World Congress, pages 9978-9983, August 2011.

[12] R. Hildebrand and M. Gevers. Central extensions in closedloop optimal experiment design. In 52nd IEEE Conference on Decision and Control, pages 5822-5827, December 2013.

[13] R. Hildebrand, M. Gevers, and G. Solari. Closed-loop optimal experiment design: Solution via moment extension. IEEE Transactions on Automatic Control, 2014. Submitted.

[14] R. Hildebrand, M. Gevers, and G. Solari. Closed-loop optimal experiment design: The partial correlation approach. In Decision and Control (CDC), 2010 49th IEEE Conference on, pages 2855-2862, Dec.

[15] Håkan Hjalmarsson. System identification of complex and structured systems. In European Control Conference, pages 3424-3452, Budapest, Hungary, August 2009.

[16] Henrik Jansson. Experiment design with applications in identification for control. PhD thesis, KTH, Stockholm, 2004.

[17] Lennart Ljung. System Identification: Theory for the User, 2nd Edition. Prentice Hall, Upper Saddle River, New Jersey, 1999.

[18] D. Materassi and G. Innocenti. Topological identification in networks of dynamical systems. Automatic Control, IEEE Transactions on, 55(8):1860 -1871, aug. 2010.

[19] B.M. Sanandaji, T.L. Vincent, and M.B. Wakin. Exact topology identification of large-scale interconnected dynamical systems from compressive observations. In American Control Conference (ACC), 2011, pages 649-656, 29 2011-july 12011.

[20] A.J. Seneviratne and V. Solo. Topology identification of a sparse dynamic network. In Decision and Control $(C D C)$, 2012 IEEE 51st Annual Conference on, pages 1518-1523, Dec 2012.

[21] Bo Wahlberg, Håkan Hjalmarsson, and Jonas Mårtensson. Variance results for identification of cascade systems. Automatica, 45(6):1443-1448, 2009.

[22] Bo Wahlberg and Cristian R. Rojas. On asymptotic frequency response variance expressions for estimated output error models. In 2012 IEEE 51st Annual Conference on Decision and Control $(C D C)$ ), IEEE Conference on Decision and Control. Proceedings, pages 178-183. IEEE, 2012.

[23] Ye Yuan, Guy-Bart Stan, Sean Warnick, and Jorge Goncalves. Robust dynamical network structure reconstruction. Automatica, 2011. 